\title{
Software Metric and Fault Prediction Using Hybrid FISHER Filter- ANNIGMA Framework
}

\author{
Peddada Venkateswara Rao ${ }^{1}$, Dorababu Sudarsa ${ }^{2}$, Ravi Kumar Tata ${ }^{3}$, Kotakonda Madhubabu ${ }^{4}$ \\ ${ }^{1,2,4}$ Asst. Professor, Department of Computer Science and Engineering, Koneru Lakshmaiah Education \\ Foundation, Vaddeswaram, AP, India. \\ ${ }^{3}$ Associate Professor, Department of C.S.E, Koneru Lakshmaiah Education Foundation, Vaddeswaram, AP, \\ India. \\ 1 pvrao.pd@gmail.com, ${ }^{2}$ dorababu.sudarsa@gmail.com, ${ }^{3}$ rktata@kluniversity.in, ${ }^{4}$ madhubabu@kluniversity.in
}

\begin{abstract}
Software Quality is one of the biggest assets in the Software development process. Any projects success or failure is dependent on the Software Quality. Software Quality can be assured through various phases of testing [1]. Testing techniques like alpha-beta testing, Black box testing and White box testing techniques can assure the software quality to a certain level. Faults can be predicted and rectified by these techniques. Manually testing can be very expensive and sometime impossible to perform testing. An automated hybrid software fault prediction model that is capable to measure the relative quality of software and identify their faulty components can significantly reduce software development effort and the threat of software project failure. In our paper we used a hybrid algorithm using ANN(Artificial Neural Networks) Wrapper and FISHER Filter techniques.
\end{abstract}

Key words : ANN Wrapper, FISHER Filter, MR Filter, Defect prediction.

\section{INTRODUCTION}

The considerable amount of money spending on software testing gives its crucial role being played in the software enterprise. However, the modern programming style in software systems are large and complex which makes it challenging in finding out the defects and selection of the best metrics to be used when predicting defects. Also, continuous upgradations of processors, operating systems make it hard to predict these defects so, overcoming these complexities is very challenging. Since for large and complex program structures there must be a significant number of test cases where they are too expensive and tedious. Manual prediction of software defects can lead to inaccuracies and further complexities, one of the following paper[16] showed this, in order to avoid those circumstances, it is better to use automated software fault detection strategies which provide accurate results.

Commonly, to understand the level of software reliability we use software quality measures mainly internal and external metrics. The internal metrics are used to measure the source code whereas external metrics to predict the behavior and functionalities of the software. Major changes in the key attributes of different entities can indicate problems in design, quality, and reliability.

Many of the automated defect-prone strategies are in high importance for dynamic software systems in the course of software development processes[21]. Many of the automated approaches use machine learning (ML) techniques such as classification algorithms, k-means clustering, Naïve Bayes (NB), support vector machines (SVM), and neural networks as an internal metric for software defect prediction. [15] [17] [18] these papers covered how efficiently defect detection can be handled.

Prior work to the researchers combines both the filter and wrapper approaches to produce a better metric selection. Filter models use heuristic functions on the datasets to reduce the overall search space. However, these filter models do not consider their performance evaluation [13] during metric ranking process is done. Therefore, using filters can give poor performance consuming a large amount of time and sometimes inefficient in fault prediction of a software system. On the other hand, wrappers use an induction algorithm to predict the performance of a selected subset.

The research of previous authors proposed a hybrid wrapper-filter approach for software defect prediction and metric selection. The two wrapper-based hybrid models include Support Vector Machines (SVM) and Artificial Neural Networks (ANN) heuristics, and then these models are used to find the defects in a software system and helps to choose the best metric for the respective framework.

The key contributions In the Hybrid Framework (Sequential) are as follows:

1. Defect prediction and key metric selection problem are combined as a single optimization problem.

2. Uses a hybrid model that combines SVM and ANN with maximum relevance (MR) filter for predicting high accuracies. 
Peddada Venkateswara Rao et al., International Journal of Emerging Trends in Engineering Research, 8(9), September 2020,5357 - 5361

3. It checks the performance of the hybrid algorithm using the filter score.

Since the above proposed cross breed structure sets aside an excessive amount of effort to complete their handling and is to be considered as a key issue in this advanced period of PC design, one approach to conquer this restriction is to utilize a parallel forecast framework for bridling the full intensity of the PC engineering. To improve the speed various frameworks are associated through a rapid system to execute them in a parallel forecast, so the determination of indicator must be planned carefully[22].

\section{BACKGROUND AND RELATED WORK}

A systematic review, primarily intended for software fault prediction (SFP) using ML techniques, of 64 primary studies with seven categories from January 1991 to October 2013, was presented in [5]. ML models are superior to the traditional statistical models to classify software modules as either defective or non-defective. It was identified in the study that; the most used metric selection technique is the alter approach of correlation-based feature selection and most frequently used metrics are the procedural metrics

The most useful OO metrics are CBO, RFC, and LOC and the most frequently used dataset is the NASA dataset.

A systematic literature review was carried out in [12] on 106 primary studies to determine which are the most widely used metrics for software defect prediction. It is observed from the study that $49 \%$ of the metrics used are OO (Chidamber and Kemerer (CK) metrics are among the highest), $27 \%$ are traditional source code metrics or $24 \%$ are process metrics.

Table 1: Comparison of existing work

\begin{tabular}{|l|l|l|}
\hline $\begin{array}{l}\text { Approach } \\
\text { name }\end{array}$ & $\begin{array}{l}\text { Significant } \\
\text { contribution }\end{array}$ & Limitations \\
\hline Filter & $\begin{array}{l}\text { Computationally } \\
\text { faster }\end{array}$ & $\begin{array}{l}\text { Does not evaluate } \\
\text { the subset }\end{array}$ \\
\hline Wrapper & $\begin{array}{l}\text { Good prediction } \\
\text { performance }\end{array}$ & $\begin{array}{l}\text { A potential risk of } \\
\text { over-fitting }\end{array}$ \\
\hline $\begin{array}{l}\text { Greedy } \\
\text { Forward } \\
\text { selection }\end{array}$ & $\begin{array}{l}\text { Uses Swarm } \\
\text { Optimization }\end{array}$ & $\begin{array}{l}\text { Makes Search } \\
\text { space wider } \\
\text { compared to filter }\end{array}$ \\
\hline ANFIS & Fuzzy Membership & Only classification \\
\hline $\begin{array}{l}\text { Random } \\
\text { forest }\end{array}$ & $\begin{array}{l}\text { Uses method level } \\
\text { metric }\end{array}$ & $\begin{array}{l}\text { No Significant } \\
\text { improvement in } \\
\text { approach and no } \\
\text { feature selection }\end{array}$ \\
\hline Naïve Bayes & $\begin{array}{l}\text { Uses method level } \\
\text { Metrics }\end{array}$ & $\begin{array}{l}\text { No Significant } \\
\text { improvement in } \\
\text { approach and no } \\
\text { feature selection }\end{array}$ \\
\hline $\begin{array}{l}\text { Defect- } \\
\text { proneness }\end{array}$ & $\begin{array}{l}\text { A feature selection } \\
\text { framework uses } \\
\text { existing classifier }\end{array}$ & $\begin{array}{l}\text { Two separate steps } \\
\text { for feature } \\
\text { selection and } \\
\text { classification and } \\
\text { similar search } \\
\text { strategy of filter } \\
\text { approach }\end{array}$ \\
\hline
\end{tabular}

Other comparisons are shown in Table 1.In the proposed framework a combination of filter and wrapper is used.

\section{FILTERSS AND WRAPPERS}

Filter [4] and wrapper [3] methods are useful for the selecting the key metrics. Filter analyses the intrinsic properties of the metrics, then the evaluation by the prediction model, to score the selected subset of metrics and to determine the best subset of metrics. Filters are computationally fast as there is no need to run an algorithm for scoring the subset of metrics. But in cases filters may predict redundant metrics which results in less performance.

Wrapper applies prediction algorithm $2^{\mathrm{n}}$ times to select the best metric subsets from the $\mathrm{n}$-dimensional metrics. This method is computationally expensive. Wrapper method provides good prediction performance due to having interactions with the subset elements. It has a potential risk of overfitting. There are other techniques such as Greedy forward selection, threshold moving, ANFIS, Naïve Bayes, Random forest, Defect Proneness which has their own significance and Limitation. The significance of Greedy Forward Selection is Uses Swarm optimization - Key feature selection by quantum particle swarm optimization (QPSO) technique and applying these features to an ANN based classifier to detect software fault-proneness.

Threshold moving technique is applied at the end of a boosting algorithm to reduce the effect of misclassification. The limitation is No feature selection

\section{DRAWBACKS IN SEQUENTIAL MR-FILTER AND ANN-WRAPPER}

\subsection{MR-FILTER:}

MR heuristic can select salient features in data mining area. It uses mutual information which statistically summarizes the degree of relevance. Significant metrics provide more relevant information about the class variable than the insignificant metrics. So, MR-filter could be applied to select the significant metrics which are more relevant to the class variable, by means of using statistical heuristics.

In Sequential Hybrid MR-Filter and ANN-Wrapper, there are back ward elimination methods and MR-Filter does not give hundred percent performance. No filter can give complete performance but using fisher we can avoid back ward elimination steps in the algorithm that reduces algorithm complexity and improves performance. This can be done in both serial and parallel methods. In previous works[10] they used SVM-Wrapper and a BE process is engaged, but in $\operatorname{SVM}$ (Support Vector Machines) [2], the main drawback is to identify a kernel for a given data set which is very difficult and it does not give accurate results for large data.

\subsection{FISHER-FILTER}

In FISHER filter [12], a subset of features are clustered in a way that the distance between different data points in 
Peddada Venkateswara Rao et al., International Journal of Emerging Trends in Engineering Research, 8(9), September 2020,5357 - 5361

different classes becomes as far as possible and in the data points in the same class becomes as close as possible for the data domain of the selected features.

Let us consider that there are distinct classes

ck $\in \mathrm{C}$

with sample size ${ }^{-}$sk, $\mathrm{k}=1,2, . . \mathrm{d}$

Here $\mu \mathrm{k}$ is the mean and $\sigma \mathrm{k}$ is the standard deviation, of $\mathrm{kth}$ class.

Calculating FISHER score:

$\operatorname{FISHER}_{(\mathrm{PI})}=\sum_{\mathrm{k}=1}{ }^{\mathrm{d}} \mathrm{s}_{\mathrm{k}}\left(\mu_{\mathrm{k}}-\mu\right)^{2} /(\sigma)^{2}$

Where $(\sigma)^{2}=\sum_{k=1}{ }^{d} S_{k}(\sigma)^{2}$

\section{PROBLEM STATEMENT}

Here there are a set of $X$ training software modules $Y,\left\{Y: y_{j} \mid\right.$ $j=1,2,3, \ldots, X\}$ which have the corresponding set $C$ of fault/no-fault classes $\left\{C: c_{l} \mid l=1,2,3, \ldots, X\right\}$. we have a set of $\mathrm{x}$ metrics $\left\{P: p_{i} \mid i=1,2,3 \ldots, x\right\}$ based on that, the faults are identified in $Y$. The problem of determining the set of significant software metric mathematically can be defined as

$\mathrm{G}(P, Y)=\operatorname{argmax} \operatorname{Perf}(S k \mid Y)$

Here $\operatorname{Perf}(S k \mid Y)$ is a performance function that determines the capability of a set of metrics $S k$ to identify the software fault which can be defined as

$\operatorname{Perf}(S k \mid \mathrm{Y})=\Sigma \mathrm{y}^{\wedge} \mathrm{i} / \mathrm{X}$

where $y^{\wedge} i=1$ if the predicted fault type $c^{\wedge} i$ by the decision function $\mathrm{D}$ of the wrapper is equal to the actual type $c i$ otherwise $\hat{y} i=0$.

One of the most important challenge is to find which wrapper will maximize the performance function $\mathrm{F}$.

Here, eq 1 is computationally expensive task. Using different filter, we can reduce the

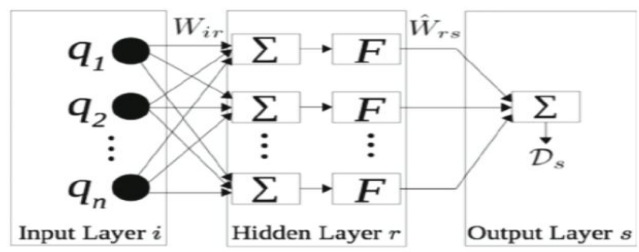

Fig. 1 Input layer, hidden layer and output layers of a multi-layer perceptron. [10]

complexity. But only to a certain level complexity can be reduced.

\section{PROPOSED FRAMEWORK}

Hybrid Framework using FISHER-Filter and ANN Wrapper: The Wrapper proposed in our framework is Artificial Neural Network Based Gain Measurement Approximation (ANNIGMA) [6]. Maximum performance is guaranteed using this wrapper because evaluation is done for each subset
$\mathrm{S}_{\mathrm{k}}$. This may improve the performance but increases the search space complexity as there are no BE steps involved.

Algorithm1 uses ANNIGMA Wrapper and Fisher-Filter. The input of this Algorithm will be $\mathrm{N}$ which will initially be True.

Decision function:

$D s=\sum r F\left(\sum r p i * N i r\right) * N r s$

Here, i, r, s are input layer, hidden layer and output layer. $\mathrm{N}_{\mathrm{ir}}$ and $\mathrm{N}_{\mathrm{rs}}$ are weights is the Neural network. From Fig.2

ANNIGMA Score is calculated. The calculation is as follows:

$\operatorname{ANNIGMA}\left(\mathrm{p}_{\mathrm{is}}\right)=\mathrm{LG}_{\text {is }} / \max \mathrm{LG}_{\mathrm{ns}}$

Where LG is Local Gain

$\mathrm{LG}=\sum_{\mathrm{r}}\left|\mathrm{N}_{\mathrm{ir}}{ }^{*} \mathrm{~N}_{\mathrm{rs}}\right|$

Average ANNIGMA score of all the folds should be calculated.

$\operatorname{ANNIGMA}\left(\mathrm{p}_{\mathrm{is}}\right)_{\text {average }}=1 / \mathrm{x} \sum \mathrm{ANNIGMA}\left(\mathrm{p}_{\mathrm{is}}\right)_{\text {fold }}$

$\operatorname{ANNIGMA}\left(\mathrm{p}_{\mathrm{i}}\right)=1 / \mathrm{k} \sum \operatorname{ANNIGMA}\left(\mathrm{p}_{\text {is }}\right)_{\text {average }}$

$\mathrm{K}$ is the total no of output nodes.

FISHER-ANNIGMA $=\operatorname{FISHER}\left(\mathrm{p}_{\mathrm{i}}\right)+\operatorname{ANNIGMA}\left(\mathrm{p}_{\mathrm{i}}\right)$

The fisher filter score $\mathrm{p}_{\mathrm{i}}$ is calculated from equation (3).

Algorithm1: Hybrid FW using FISHER-FILTER

1. Input $\left\{Y: Y_{j} \mid j=1,2,3 \ldots X\right\}$ data from software modules with $\mathrm{n}$ metric

2. Input $\mathrm{N} \leftarrow$ True for $\mathrm{ANN}$

3. Output $\leftarrow$ SBEST significant metrics

4. $S \leftarrow y_{j}$ whole set of $x$ metrics

5. S0 $\leftarrow$ initial set of metrics

6. Select the FISHER- FILTER

7. For $\mathrm{i}=0$ to $\mathrm{n}-1$ do

8. Compute Fisher score using (3)

9. For fold $=1$ to $\mathrm{x}$ do

10. Train the wrapper ANN with software metric

11. set Scurrent

12. Compute ANNIGMA scores of all metrics in the set Scurrent using equation (4)

13. Compute accuracy

14. End for

15. Compute average ANNIGMA of Scurrent using equation (5)

16. Compute average ANNIGMA of one-fold using equation (6) 
Peddada Venkateswara Rao et al., International Journal of Emerging Trends in Engineering Research, 8(9), September 2020,5357 - 5361

17. Rank the metrics in descending order

18. Combine the current metrics with the initial metrics $\mathrm{S} 0=\mathrm{S} 0 \mathrm{U}$ Scurrent

19. End for

20. Identify the best metrics with highest accuracy

21. Return best metric Sbest

\section{EXPERIMENT RESULT}

In this paper we can evaluate our algorithm by taking different NASA data sets available, but here we have taken PC3 data set for performance analysis. Here we compare our Hybrid Model with the Maximum relevance and ANNIGMA algorithms. A MEWMA(Multivariate exponentially weighted moving average)[20] is used to continuously monitor and measure the performance of two or more characteristics of any output control situation. The following Table. 2 shows our Hybrid framework achieved $94.5 \%$ in control vs. $4 \%$ as out of control situation and $93 \%$ vs. $5.6 \%$ for in control vs.4\% as out of control situation, respectively.

Table 1: MEWMA results for software fault prediction dataset PC3

\begin{tabular}{|l|l|l|l|}
\hline Characteristics & $\begin{array}{l}\text { maximu } \\
\text { m } \\
\text { relevance }\end{array}$ & $\begin{array}{l}\text { ANNIGM } \\
\text { A }\end{array}$ & $\begin{array}{l}\text { Hybrid } \\
\text { Model }\end{array}$ \\
\hline Total Points & 1277 & 1277 & 1277 \\
\hline Total OC Points & 200 & 70 & 50 \\
\hline Total IC Points & 750 & 1200 & 1244 \\
\hline Percentage(IC) & $75 \%$ & $93 \%$ & $94.5 \%$ \\
\hline Percentage(OC) & $19 \%$ & $5.6 \%$ & $4 \%$ \\
\hline
\end{tabular}

Therefore, we can come to a conclusion that our Hybrid model is best compare to other models.

\section{CONCLUSION}

In this paper we discussed about the most important part of Fault prediction and rectification process. Most of the Legacy models are not reached up to expected level. To overcome the problems mentioned in this paper, we proposed hybrid software fault prediction model that is capable to measure the relative quality of software and identify their faulty components can significantly reduce software development effort and the threat of software project failure. In our paper we used a hybrid algorithm using ANN(Artificial Neural Networks) Wrapper and FISHER Filter techniques. Using Machine learning techniques for software defect detection $[11],[19]$ is the feature scope of this field.

\section{REFERENCES}

1. F. P. Seth, O. Taipale and K. Smolander, "Organizational and customer related challenges of software testing: An empirical study in 11 software companies", 2014 IEEE Eighth International Conference on Research Challenges in Information
Science (RCIS), Marrakech, 2014, pp. 1-12, doi: 10.1109/RCIS.2014.6861031.

2. N. Cristianini and J. Shawe-Taylor, "An Introduction to Support Vector Machines and Other Kernel-Based Learning Methods". New York, NY, USA: Cambridge Univ. Press, 2000.

3. J. A. Rodger, "Toward reducing failure risk in an integrated vehicle health maintenance system: A fuzzy multi-sensor data fusion Kalman filter approach for IVHMS', Expert Syst. Appl., vol. 39, no. 10, pp. 9821-9836, Aug. 2012.

4. F. Aparisi and J. Sanz, "Interpreting the out-of-control signals of multi- variate control charts employing neural networks", Int. J. Comput., Elect., Autom., Control Inf. Eng., vol. 4, no. 1, pp. 24-28, 2010.

5. H. B. Yadavand D. K. Yadav, "A fuzzy logic-based approach for phase- wise software defects prediction using software metrics', Inf. Softw. Tech- nol., vol. 63, pp. 44-57, Jul. 2015.

6. C.-N. Hsu, H.-J. Huang, and S. Dietrich, "The ANNIGMA-wrapper approach to fast feature selection for neural nets," IEEE Trans. Syst., Man, Cybern. B, Cybern., vol. 32, no. 2, pp. 207-212, Apr. 2002.

7. R. Kohavi and G. H. John, "Wrappers for feature subset selection", Artif. Intell., vol. 97, nos. 12, pp. 273324, 1997.

8. K. S. Balagani and V.V. Phoha, "On the feature selection criterion based on an approximation of multidimensional mutual information", IEEE Trans. Pattern Anal. Mach. Intel., vol. 32, no. 7, pp. 13421343, Jul. 2010.

9. Venkata Ramana, N., Kolli, C. S., Ravi Kumar, T., \& Nagesh, P. (2019). "Hybrid K-Mir algorithm to predict type of lung cancer among stoicism", International Journal of Innovative Technology and Exploring Engineering, 8(4), 283-287.

10. Huda, Shamsul, et al. "A framework for software defect prediction and metric selection." IEEE access 6 (2017): 2844-2858.

11. Tata, R.K., Mothe, S. , Koneru, P., Venkata Ramana, N. , Sadhana, B., "Load balancing analyzer: A recommendation system using machine learning", International Journal of Emerging Trends in Engineering Research, 2020, 8(5), pp. 2085-2090.

12. D. Radjenoviф, M. Herio, R. Torkar, and A. Sivkovi, "Software fault prediction metrics: A systematic literature review", Inf. Softw. Technol., vol. 55, no. 8, pp. 13971418,2013.

13. Krishna, T. Lakshmi Siva Rama, and Thirumalaisamy Ragunathan. "Performance evaluation of speculative semantics-based algorithm for read operations in distributed file system." International Journal of Communication Networks and Distributed Systems 22, no. 3 (2019): 275-293. 
14. Krishna, B. Chaitanya, Kodukula Subrahmanyam, and Tai-hoon Kim. "A dependency analysis for information security and risk management." International Journal of Security and Its Applications 9.8 (2015): 205-210.

15. Prasanth Y., Sreedevi E., Gayathri N., Rahul A.S. (2017),"Analysis and implementation of ensemble feature selection to improve accuracy of software defect detection model ", Journal of Advanced Research in Dynamical and Control Systems,9(18 Special Issue),PP.601-613.

16. Hussain M.A., Kiran Kumar K., Venkata Avinash J., Vinay Sai Kumar P. (2018)," Implementation of software application for employee's health monitoring system", Journal of Advanced Research in Dynamical and Control Systems, 10 (),PP. 445-451

17. Sreedevi E., Prasanth Y. (2019), "A novel ensemble feature selection and software defect detection model on promise defect datasets", International Journal of Recent Technology and Engineering, 8(1), PP.3131-3136.

18. Sreedevi E., Premalatha V., Sivakumar S., Nayak S.R. (2019), "A comparative study on new classification algorithm using NASA MDP datasets for software defect detection", Proceedings of the International Conference on Intelligent Sustainable Systems, ICISS 2019, (), PP.312-317.

19. Venkata Raghava Rao Y., Burri R.D., Prasad V.B.V.N. (2019), "Machine learning methods for software defect prediction a revisit.", International Journal of Innovative Technology and Exploring Engineering, 8(8), PP.3431-3435.

20. H. Hashimoto et al., "Epileptic seizure monitoring by using multivariate statistical process control", in Proc. 12th IFAC Symp. Comput. Appl. Biotechnol., Int. Fed. Autom. Control, 2013, pp. 16-18.

21. Shahabuddin S.M., Yalla P. (2017),'Impact of lean software development into agile process model with integration testing prior to unit testing", Journal of Theoretical and Applied Information Technology,95(22),PP.6163-6175.

22. Malleswari D.N., Sowmya T.S., Mukhul K., Reddy D. (2018),"Analysis on software project planning and control using case tools", International Journal of Engineering and Technology(UAE),7 (0),PP. 158-160 